\title{
Synergistic antineoplastic action of 5-aza-2'deoxycytidine (decitabine) in combination with different inhibitors of enhancer of zeste homolog 2 (EZH2) on human lung carcinoma cells
}

\author{
Antônia Sâmia Fernandes do Nascimento ${ }^{1}$, Sylvie Côté ${ }^{1}$, Lak Shin Jeong ${ }^{2}$, Jinha Yu² and Richard L Momparler ${ }^{1,3, *}$ \\ 1 Centre de recherche, Service d'hématologie/oncologie, CHU-Saint-Justine, Montréal, Québec H3T 1C5, Canada \\ 2 Research Institute of Pharmaceutical Sciences, College of Pharmacy, Seoul National University, Seoul 151-742, Korea \\ ${ }^{3}$ Département de Pharmacologie, Université de Montréal, Montréal, QC H3T 1J4, Canada
}

\begin{abstract}
Patients with metastatic lung cancer have a very poor prognosis indicating an urgent need to develop more effective chemotherapy. Aberrant promoter DNA methylation can result in the epigenetic silencing of tumor suppressor genes (TSGs) in lung cancer. 5-Aza2'deoxycytidine (5-Aza-CdR, decitabine), an inhibitor of DNA methylation, is able to reactivate silent TSGs. Trimethylation of histone $\mathrm{H} 3$ on lysine $27(\mathrm{H} 3 \mathrm{~K} 27 \mathrm{me} 3)$ by enhancer of zeste homolog $2(\mathrm{EZH} 2)$ histone methyltransferase can also silence TSGs in lung cancer. 3-Deazaneplanocin-A (DZNep), an inhibitor of EZH2, up-regulates the expression of genes silenced by H3K27me3. In this study we compared the in vitro antineoplastic activity of different inhibitors of EZH2; DZNep, U-4149 and Gsk-126, alone and in combination with 5-Aza-CdR, on the human A549 lung adenocarcinoma cells. U-4149, an analogue of DZNep, was more potent than either DZNep or Gsk126. The reduction in colony formation was dose- and time-dependent for each EZH2 inhibitors. Combination treatment of 5-Aza-CdR with the EZH2 inhibitors showed a synergistic antineoplastic activity. 5-Aza-CdR and U-4149 was the most potent combination. The in vitro antineoplastic activity of these agents was evaluated by inhibition of growth, colony formation, induction of senescence and apoptosis. All the drug combinations induced signs of senescence and apoptosis. Analysis by gene expression by qRT-PCR showed that the combinations increased the expression of several TSGs to a greater extent that either agent alone. In conclusion, epigenetic therapy that specifically targets DNA and histone methylation has interesting potential for the treatment of lung cancer and merits further investigation.
\end{abstract}

Keywords: 5-aza-2'deoxycytidine; decitabine; 3-deazaneplanocin-A; Gsk-126; U-4149; lung cancer; antineoplastic action; epigenetics; DNA methylation; EZH2

\section{Introduction}

Lung cancer is a leading cause of cancer-associated mortality worldwide; the most common type is non-small cell lung cancer (NSCLC) [1, 2]. Clinical and molecular studies indicate that both genetic and epigenetic events are implicated in tumorigenesis of NSCLC [3]. It is not possible to reverse the genetic aberrations with current chemotherapy. However, epigenetic changes are potentially reversible and are promising targets for chemotherapeutic intervention [4]. Two of the key epigenetic alterations that are observed in NSCLC are aberrant DNA and histone methylation $[5,6]$.

DNA hypermethylation is an epigenetic modification that leads to the transcriptional silencing of regulatory genes, including tumor suppressor genes (TSGs). The silencing of these genes can promote tumorigenesis by several mechanisms including increasing cell proliferation and inactivation of genes thatsuppress metastasis, angiogenesis and apoptosis [7, 8]. Several TSGs were reported to be silenced by DNA methylation in NSCLC, which correlated with poor survival $[9,10]$. The expression of these genes can be restored by a potent inhibitor of DNA methylation,
5-Aza-2'deoxycytidine (5-Aza-CdR/decitabine) [11, 12]. The action of 5-Aza-CdR results from its incorporation into DNA, resulting in the loss of DNA methylation by irreversibly trapping the DNA methyltransferase 1 (DNMT1) to DNA [13].

The potential clinical importance of inhibitors of DNMT1 in lung cancer is supported by some interesting responses observed in some NSCLC patients treated with 5-Aza-CdR

*Corresponding author: Richard L Momparler, Ph.D., Centre de recherche, CHU-Saint-Justine, Montréal, Québec H3T 1C5, Canada. Tel.: 1-514-345-4931 ext 6140; Fax: 1-514-345-4801; Email: richard.I.momparler@umontreal.ca

Received 25 June 2016 Revised 16 August 2016 Accepted 12 September 2016 Published 23 September 2016

Citation: Nascimento ASF, Côté S, Jeong LS, Yu J, Momparler RL. Synergistic antineoplastic action of 5-aza-2'deoxycytidine (decitabine) in combination with different inhibitors of enhancer of zeste homolog 2 (EZH2) on human lung carcinoma cells. J Cancer Res Ther. 2016; 4(5):42-49. DOI: 10.14312/2052-4994.2016-8

Copyright: (c) 2016 Nascimento ASF, et al. Published by NobleResearch Publishers. This is an open-access article distributed under the terms of the Creative Commons Attribution License, which permits unrestricted use, distribution and reproduction in any medium, provided the original author and source are credited. 
alone or 5-azacytidine in combination with an inhibitor of histone deacetylase (HDACs) [14, 15]. These reports provide a rationale to search for other agents to use with 5-AzaCdR to increase its efficacy for the treatment of NSCLC. Another interesting aspect of DNA methylation inhibitors is their "epigenetic priming", which can make tumors more sensitive to subsequent treatment with cytotoxic drugs $[16,17]$.

A second epigenetic pathway that has been shown to be dysregulated in cancer is gene silencing mediated by the polycomb repressor complex 2 (PRC2) [18, 19]. EZH2 (enhancer of zeste homolog 2), the catalytic subunit of $\mathrm{PRC2}$, is a histone methyltransferase that trimethylates lysine 27 in histone H3 (H3K27me3), an epigenetic marker that silences gene expression. Deregulation of $\mathrm{EZH} 2$ is proposed to occur during malignant transformation in different types of cancer [20-22]. EZH2 is frequently overexpressed in NSCLC and is correlated with poor prognosis [23]. These observations suggest that EZH2 is an interesting target for chemotherapy of lung cancer.

3-Deazaneplanocin-A(DZNep) is a S-adenosylhomocysteine hydrolase inhibitor that inhibits EZH2 activity and induces apoptosis in different types of cancer cells [24-26]. DZNep also shows significant antineoplastic activity against NSCLC cells [27]. Other agents have been developed that also target EZH2. U-4149 is an analogue of DZNep with a similar mechanism of action. Gsk-126 is a specific inhibitor of EZH2 and currently is clinical trial in patients with lymphoma (ClinicalTrials.gov identifier: NCT02082977).

The aim of our investigation was to compare the in vitro antineoplastic activity of DZNep, U-4149 and Gsk-126 on human A549 lung carcinoma cells. Previous studies in our laboratory have shown that 5 -Aza-CdR in combination with DZNep exhibits synergistic antineoplastic action on leukemic cells [28]. Our second aim was to investigate the relative antineoplastic activity of the different agents that target $\mathrm{EZH} 2$ in combination with $5-\mathrm{Aza}-\mathrm{CdR}$ against the lung carcinoma cells.

\section{Materials and methods}

\section{Cell lines and materials}

Human non-small cell lung cancer cell line A549 (adenocarcinoma) was purchased from American Type Culture Collection (ATCC). A549 was cultured in $\alpha$-MEM medium (Invitrogen) with 10\% fetal bovine serum (FBS) and maintained in a $37^{\circ} \mathrm{C}$ incubator with $5 \% \mathrm{CO}_{2} .5$-Aza$\mathrm{CdR}$ was obtained from the Institute of Organic Chemistry (Prague, Czech Republic,). DZNep (3-deazaplanocin-A) was kindly provided by Dr Victor E. Marquez, Chemical Biology Laboratory (Frederick, MD). The chemical synthesis of U-4149, fluoro analogue of DZNep, was performed using the method of Choi et al. [29]. 5-Aza-CdR, DZNep and U-4149 were dissolved in sterile phosphate buffer saline $\mathrm{pH} 6.8$ (PBS) solution. Gsk-126 was obtained from Xcess Biosciences Inc. and was dissolved in DMSO. High Capacity cDNA Reverse Transcription Kit, random primers and universal PCR mixture were obtained from Applied Biosystems and RNeasy plus mini kit from Quiagen. Senescence staining kit was obtained from Sigma-Aldrich and CellEventR Caspase 3/7 Green Detection Reagent was obtained from Life technologies.

\section{Clonogenic assays}

The exponentially growing A549 lung carcinoma cells were trypsinized into a single cell suspension. Two hundred fifty cells were seeded in 6-well $\left(9.6 \mathrm{~cm}^{2} /\right.$ well) plates containing $\alpha$-MEM with $10 \%$ FBS. The next day the cells were treated with different concentrations of DZNep, Gsk126 and $U-4149$ for 6, 12, 24, 48 and $72 \mathrm{~h}$ using a time- and dose-response studies. At the end of treatment, the cells were suspended in drug-free medium and maintained in a $37^{\circ} \mathrm{C}$ incubator with $5 \% \mathrm{CO}_{2}$. The number of colonies after staining with Giemsa/methanol was counted after 16-18 days of incubation and the loss of clonogenicity was determined. For sequential treatment in the drug combination assay, A549 cells were treated with $0.1 \mu \mathrm{M}$ 5-Aza-CdR. At $24 \mathrm{~h}$ either $1 \mu \mathrm{M}$ DZNep, $2.5 \mu \mathrm{M}$ Gsk-126 or $1 \mu \mathrm{M}$ U-4149 were added to the medium. The cloning efficiency was in the range of $60-70 \%$. All the experiments were performed in triplicate. Similar conditions were used for the growth inhibition assay, except at each time point, the medium was aspirated and the cells trypsinized for a cell count using the Beckmann Model Z Coulter.

\section{Senescence-associated- $\beta$-galactosidase (SA- $\beta$-Gal) staining}

The senescence staining kit was used to identify senescent cells at the end of drug treatment. Twenty thousand cells were plated in 6-well plates and allowed to adhere to the surface in a $37^{\circ} \mathrm{C}$ with $5 \% \mathrm{CO}_{2}$ the day before of drug treatments. Treatments were made as described for the clonogenic assays. At $24 \mathrm{~h}$ after the end of drug treatment, the medium was aspirated and the cells were washed twice with PBS followed by incubation at room temperature with fixation buffer. The cells were rinsed with PBS and the staining mixture was added. The plate was incubated overnight at $37^{\circ} \mathrm{C}$ without $\mathrm{CO}_{2}$. The blue-stained cells (senescent) and the total number of cells were counted using a microscope. The percentage of senescent cells was compared to total number of cells.

\section{Apoptosis-associated-Caspases}

The CellEventR Caspase-3/7 Green reagent was used to detect caspases 3 and 7 in A549 tumor cells at the end of drug treatment. Ten thousand cells were seeded on glass coverslips and allowed to adhere to the surface in a $37^{\circ} \mathrm{C}$ incubator with $5 \% \mathrm{CO}_{2}$ the day before of drug treatments. Treatments proceeded as described for clonogenic assays. At $24 \mathrm{~h}$ after the end of drug treatment, the probe solution was added to the cells. The glass coverslips was incubated for $30 \mathrm{~min}$ in the dark at room temperature. Then, stained cells were observed under fluorescence microscopy.

\section{Analysis of gene expression}

For gene expression analysis, the cell treatments were performed as described in clonogenic assay. At the end of drug treatment, the cells were centrifuged, suspended in drug-free medium and counted. Total RNA purification was carried out following the manufacturer's instructions of the RNeasy plus mini kit. For cDNA preparation, $2 \mu \mathrm{g}$ of total RNA was reversed transcribed using the cDNA Reverse Transcription Kit with random primers as described by 
the manufacture. The reaction mixture contained cDNA, specific primers for the target genes (CDKN1A (p21), RUNX3 and RASSF1), Sybr green and TaqMan Fast Universal PCR Master Mix. The ABI PRISM 7900HT Sequence Detection System (Applied Biosystems) was used to detect the amplification level. All reactions were run in triplicate and the average Cts were used for quantitation. The endogenous controls were TATA-binding protein (TBP) gene and hypoxanthine-guanine phosphoribosyltransferase (HPRT) gene. The relative quantification of the target genes was determined using the $\Delta \Delta C T$ method. Briefly, the $\mathrm{Ct}$ (threshold cycle) values of the target gene were normalized to the endogenous control TPB and HPRT genes ( $\triangle \mathrm{CT}$ $=$ Cttarget -Ctcontrol) and compared with a calibrator: $\Delta \Delta C T=\Delta$ Ctsample $-\Delta$ Ctcalibrator. Relative expression (RQ) was calculated using the Sequence Detection System (SDS) 2.2.2 software (Applied Biosystems) and the formula: $\mathrm{RQ}=2-\Delta \Delta \mathrm{CT}$. The sequences of the primers used in the study were CDKN1A (Forward: tcactgtcttgtacccttgtgc and Reverse: ggcgtttggagtggtagaaa), RUNX3 (Forward: tcagcaccacaagccactt and Reverse: aatgggttcagttccgaggt) and RASSF1 A (Forward: gactctggggaggtgaactg and Reverse: ggagtacttctgcaggatctgg).

\section{Statistical analysis}

The data are expressed as means \pm standard error of mean (SEM). Statistical analyses were performed using one-way analysis of variance (ANOVA) and Student's t-test to evaluate differences between groups. $\mathrm{P}<0.05$ was considered statistically significant.

\section{Results}

Evaluation of the antineoplastic activity of DZNep, Gsk-126 and U-4149 on A549 lung tumor cells

The chemical structures of DZNep, Gsk-126 and U-4149 are shown in Figure 1. A colony assay was performed to determine the loss of clonogenicity of A549 lung tumor cells produced by each individual epigenetic agent (DZNep, Gsk126 and U-4149) at different concentrations and different exposure times. The loss of clonogenicity in A549 tumor cells was dose- and time-dependent for all the three drugs, as showed in the Figure 2. After $24 \mathrm{~h}$ exposure at $1 \mu \mathrm{M}$ DZNep, A549 tumor cells showed a loss of clonogenicity
(> 30\%) and at $2.5 \mu \mathrm{M}$ (> 40\%) (Figure 2a). For a $24 \mathrm{~h}$ exposure, $1 \mu \mathrm{M}$ Gsk-126 showed the lowest antineoplastic effect against A549 tumor cells as compared to the other EZH2 inhibitors, showing a loss of clonogenicity (>20\%) and at $2.5 \mu \mathrm{M}$ (> 30\%) (Figure 2b). The cells were more sensitive to $1 \mu \mathrm{M} U-4149$ after $24 \mathrm{~h}$ exposure, as shown by a loss of clonogenicity (> 40\%) and at $2.5 \mu \mathrm{M}$ (> 55\%) (Figure 2c). DZNep and Gsk-126 required either higher concentration or a longer exposure drugs to produce a loss of clonogenicity $>40 \%$ on A549 tumor cells.

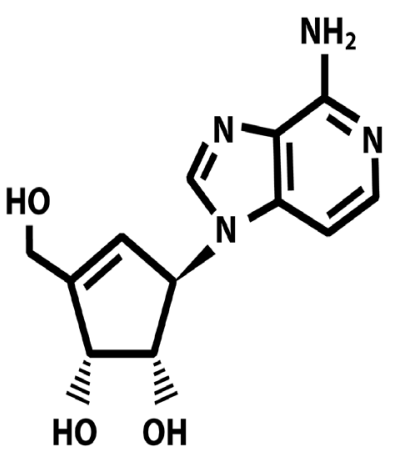

DZNep

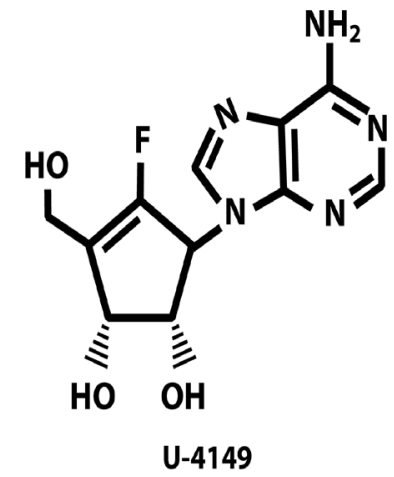

U-4149

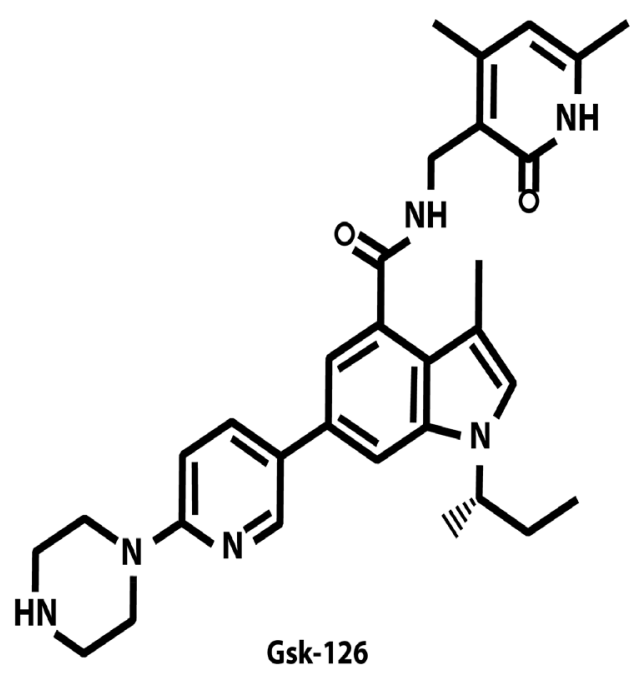

Figure 1 Chemical structures of the enhancer of zeste homolog 2 (EZH2) inhibitors: Deazaneplanocin-A (DZNep), Gsk-126 and U-4149.

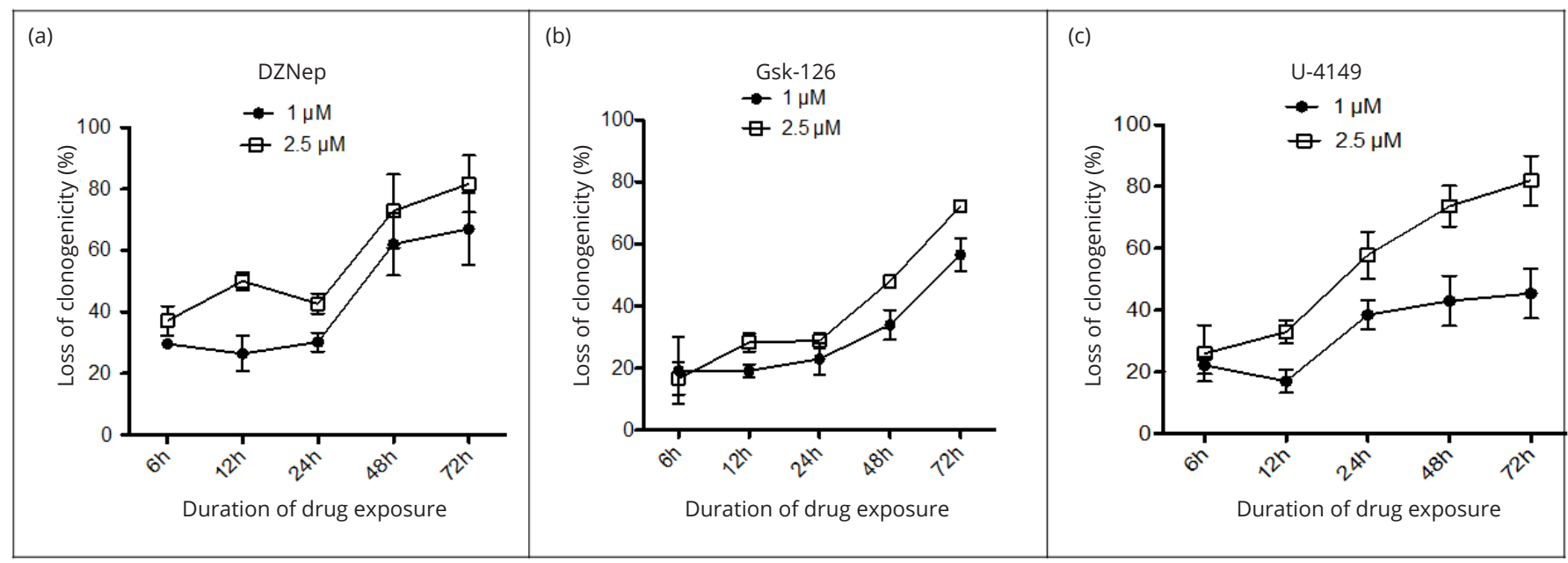

Figure 2 Time-course evaluation of the antineoplastic activity of DZNep (a), Gsk-126 (b) and U-4149 (c) on A549 cells. The cells were exposed to drugs at indicated concentrations. At different times $(6,12,24,48$ and 72 h) the drugs were removed and colony formation was determined on day $16-18$. Graphs represent the percentage of loss of clonogenicity relative to control cells. Data shown are mean values $\pm S E M, n=3$. 
Loss of clonogenicity of A549 lung tumor cells by combination of epigenetic agents

A colony assay was performed to determine the loss of clonogenicity of A549 cells produced by each individual epigenetic agent (5-Aza-CdR, DZNep, Gsk-126 and U-4149) and the different double combinations of these agents. We used a sequential drug treatment that started with 5-Aza$\mathrm{CdR}$ and was followed by the addition of the $\mathrm{EZH} 2$ inhibitors at $24 \mathrm{~h}$. The drugs were removed at $48 \mathrm{~h}$ and replaced with drug-free medium. Single agent's treatments showed the loss of clonogenicity of: $20 \%$ (5-Aza-CdR), 29\% (DZNep), $36 \%$ (Gsk-126) and 41\% (U-4149) for a $24 \mathrm{~h}$ exposure of A549 tumor cells (Figure 3a). The double combinations exhibited a significantly greater loss of clonogenicity than the single agents. The double combination 5-Aza-CdR plus DZNep showed remarkable antineoplastic activity as indicated by $53 \%$ loss of clonogenicity. 5-Aza-CdR plus Gsk126 showed $54 \%$ loss of clonogenicity and 5-Aza-CdR plus $\mathrm{U}-4149$ showed $64 \%$ loss of clonogenicity (Figure 3a). All the double combinations showed a synergistic effect against A549 tumor cells. The most synergistic effect was observed with 5-Aza-CdR plus U-4149. When the concentration of 5-Aza-CdR was increased from $0.1 \mu \mathrm{M}$ to $0.2 \mu \mathrm{M}$, all the double combinations showed a greater synergistic effect against A549 tumor cells (Figure 3b). The most synergistic effect was 5-Aza-CdR plus U4149 (> 84\%) for both concentrations of 5-Aza-CdR (0.1 or $0.2 \mu \mathrm{M})$. In conclusion, we observed that the inhibitors of EZH2, DZNep, Gsk-126 and U-4149, enhanced the action of 5-Aza-CdR on lung A549 lung tumor cells and U-4149 was more potent than DZNep or Gsk-126.

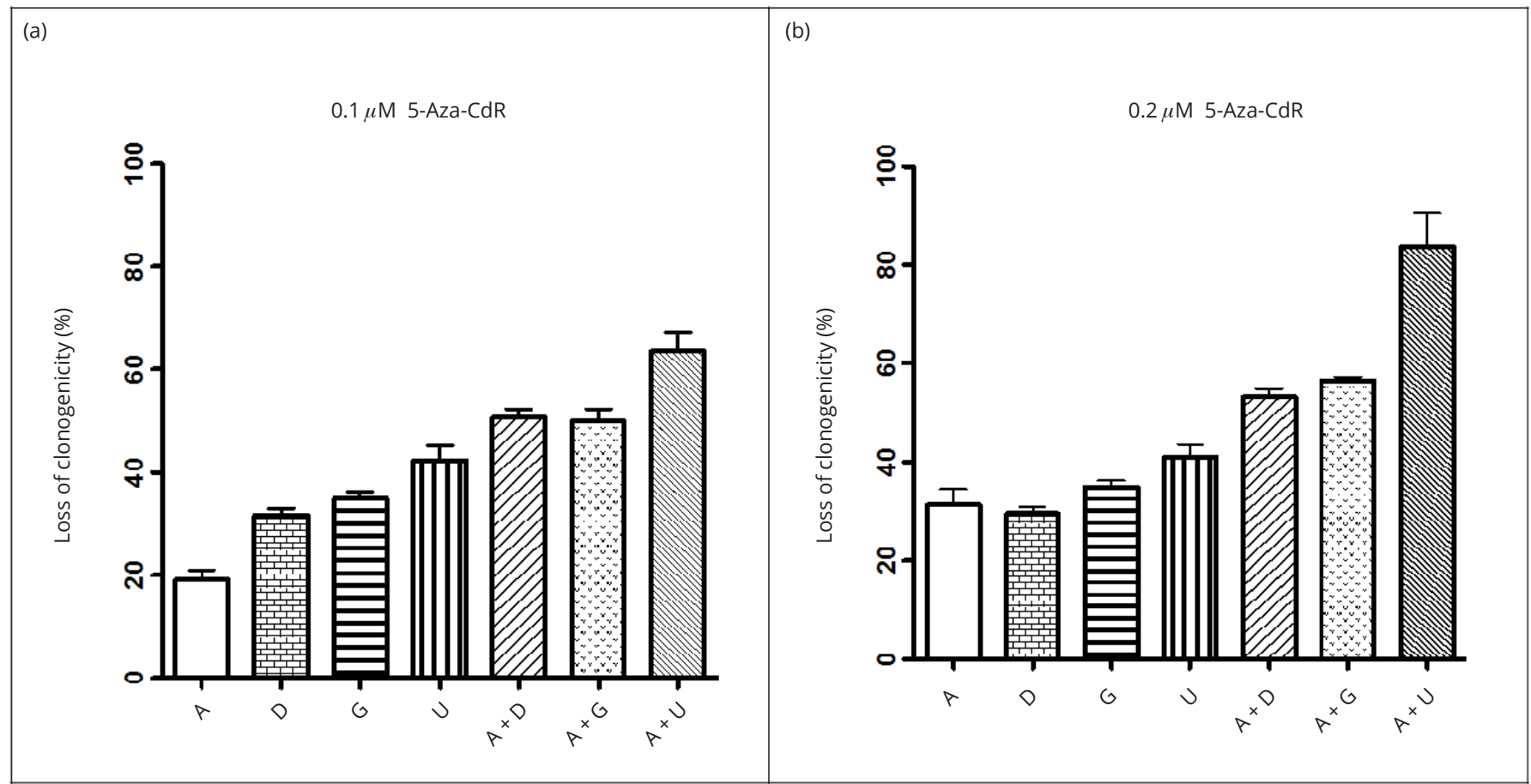

Figure 3a,b Colony assay of A549 cells after treatment with 5-Aza-CdR (A), DZNep (D), Gsk-126 (G) and U-4149 (U). Cells were treated at $0 \mathrm{~h}$ with $0.1 \mu \mathrm{M}$ 5-Aza-CdR (a) or $0.2 \mu \mathrm{M}$ 5-Aza-CdR (b) alone or in sequential combination by the addition at $24 \mathrm{~h}$ of: $1 \mu \mathrm{M}$ DZNep, $2.5 \mu \mathrm{M}$ Gsk-126 and $1 \mu \mathrm{M} \mathrm{U}-4149$. At the end of drug treatment (48 h), the cells were incubated in drug-free medium. The number of colonies was counted after 16-18 days of incubation and the loss of clonogenicity (\%) was expressed relative to control cells. Data shown are mean values $\pm S E M, n=3$.

\section{Growth inhibition assay}

The growth inhibition of A549 tumor cells was determined by cell counting at the end of drug treatment. Single agent treatment showed a growth inhibition of 33 to $35 \%$, except for $2.5 \mu \mathrm{M}$ Gsk-126, which showed only $14 \%$ growth inhibition (Table 1). All the double combinations were observed a significantly greater growth inhibition than the single agents for a total $48 \mathrm{~h}$ drug treatment (Table1).

\section{Senescence analysis}

For identifying senescent cells, we used an assay based on a histochemical stain for $\beta$-galactosidase activity. At $\mathrm{pH} 6$ this enzyme is detectable in senescent cells. Phase contrast images of A549 tumor cells after 2 days of drug treatment showed reduced cell numbers, damaged cells, and some normal-looking cells (Figure 4). A small number
Table 1 Growth inhibition assay of A549 cells after treatment with 5-AzaCdR (A), DZNep (D), Gsk-126 (G) and U-4149 (U).

\begin{tabular}{lc}
\hline Drug concentration & Growth inhibition (\%) \\
\hline 5-Aza-CdR 0.1 $\mu \mathrm{M}$ & $34.0 \pm 324 \mathrm{~h}$ \\
DZNep $1 \mu \mathrm{M}$ & $35.0 \pm 324 \mathrm{~h}$ \\
Gsk-126 $2.5 \mu \mathrm{M}$ & $14.0 \pm 324 \mathrm{~h}$ \\
U-4149 $1 \mu \mathrm{M}$ & $33.0 \pm 324 \mathrm{~h}$ \\
5-Aza-CdR + DZNep & $47.0 \pm 348 \mathrm{~h}$ \\
5-Aza-CdR + Gsk-126 & $41.0 \pm 348 \mathrm{~h}$ \\
5-Aza-CdR + U-4149 & $56.0 \pm 348 \mathrm{~h}$ \\
\hline
\end{tabular}

Note: Cells were treated with $0.1 \mu \mathrm{M}$ 5-Aza-CdR alone or in sequential combination with $1 \mu \mathrm{M}$ DZNep, $2.5 \mu \mathrm{M}$ Gsk-126 and $1 \mu \mathrm{M} \mathrm{U}-4149$. At the end of drug treatment, the cells were trypsinized and resuspended in drugfree medium and the number of cell was counted. The growth inhibition (\%) was expressed relative to control cells. Data shown are mean values \pm $\mathrm{SEM}, \mathrm{n}=3$. 
of senescent cells were identified in the untreated cells (7\%) or the cells treated with 5-Aza-CdR alone (9.5\%). The cells treated with DZNep, Gsk-126 and U-4149 showed 12\%, $9 \%$ and $16 \%$ of senescent cells, respectively. A synergistic interaction was observed for all combinations as shown by the increase in the number of senescent cells. The highest number of senescent cells was observed in 5-Aza-CdR plus
U-4149 group (100\%), followed by 5-Aza-CdR plus DZNep (24\%) and 5-Aza-CdR plus Gsk-126 (13\%). All the double combinations showed damaged cells, senescent cells and some healthy-looking cells, except for 5-Aza-CdR plus U-4149 treatment, where no healthy-looking cells were observed. The data are in accord with loss of clonogenicity and growth inhibition analysis.

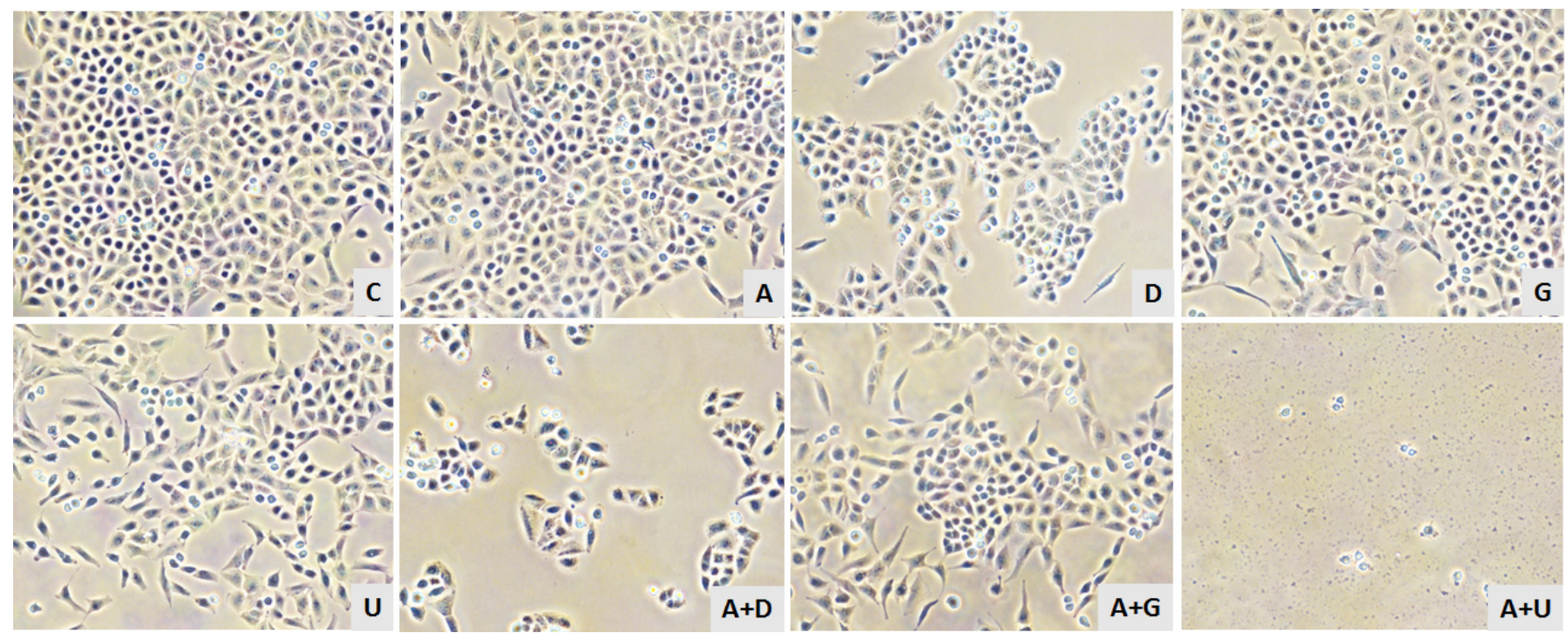

Figure 4 Effect of treatment with 5-Aza-CdR (A), DZNep (D), Gsk-126 (G) and U-4149 (U) on senescence of A549 cells. Treatment is described in Table 1. Twenty thousand cells were plated in 6-well plates and analysed for detection of senescence $24 \mathrm{~h}$ after drug treatment. The blue-stained cells (senescent) and the total number of cells were observed at microscope. The percentage of senescent cells was compared to total number of cell in each well. Original magnification $\times 400$.

\section{Apoptosis analysis}

We investigated the induction of apoptosis by 5 -Aza-CdR, DZNep, Gsk-126 and U-4149 and their combinations with 5-Aza-CdR using a caspase green detection reagent, which becomes fluorescent upon cleavage by active caspases
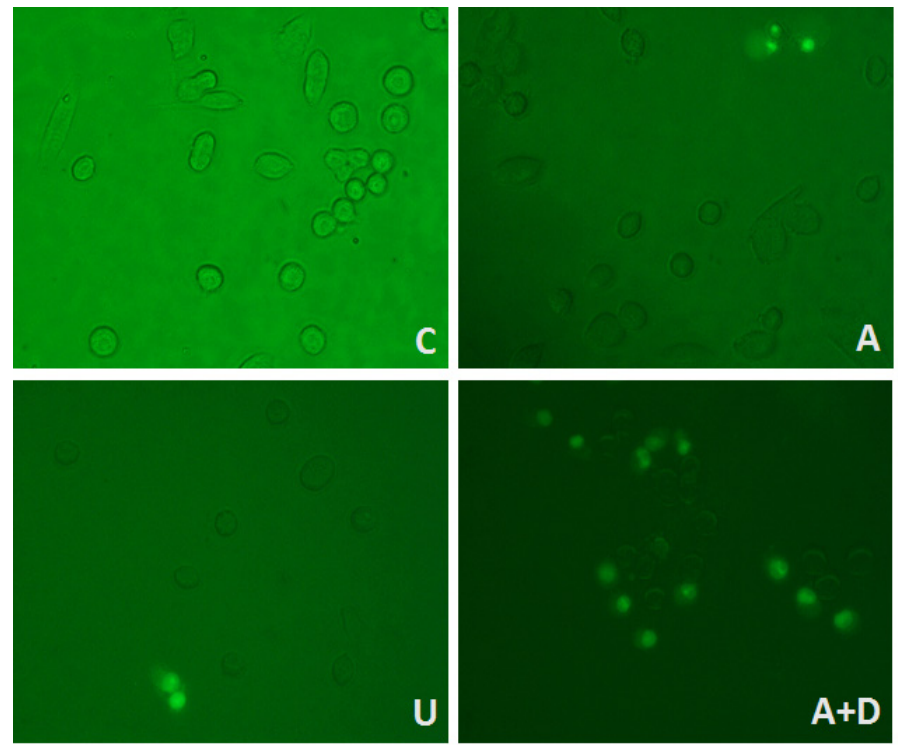

3 and 7. Our results show that these epigenetic agents induce apoptosis in A549 tumor cells and that caspases are involved in this event (Figure 5). All the combinations showed a greater number of apoptotic cells as compared to the respective single agents.
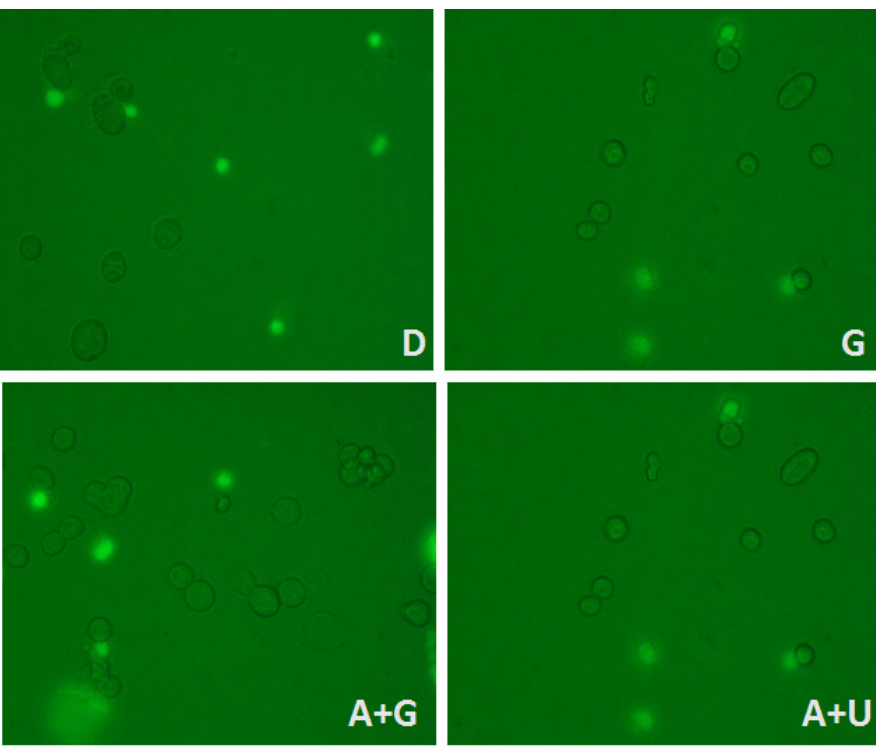

Figure 5 Detection of caspases 3 and 7 in A549 cells after treatments with 5-Aza-CdR (A), DZNep (D), Gsk-126 (G) and U-4149 (U). A549 cells were seeded on glass coverslips and allowed to adhere to the surface in a $37^{\circ} \mathrm{C}$ with $5 \% \mathrm{CO}_{2}$ the day before of drugs treatment. Treatment proceeded as described for clonogenic assays. The glass coverslips was incubated for $30 \mathrm{~min}$ in the dark at room temperature and analyzed on a fluorescent microscope. Positive apoptotic cells containing active caspases 3 and 7 appear in green. Original magnification, $\times 400$.

\section{Analysis of gene expression}

In order to understand if the antineoplastic action of these epigenetic agents correlated with the activation of genes that are frequently silenced in NSCLC, we analyzed the expression of three TSGs genes: CDKN1A (p21), RUNX3 and RASSF1. The analysis of mRNA levels of these genes 
was made by real-time RT-PCR. Each agent alone produced a small increase in expression of these genes that was less than some double combinations. 5-Aza-CdR plus DZNep produced an activation of $\mathrm{p} 21$ by $>2.0$ fold and RUNX3 by $>1.8$ fold relative to control cells. This combination did not show a significant activation of RASSF1 $(P<0.05)$ (Figure 6a). 5-Aza-CdR plus Gsk-126 produced an activation of
CDKN1A by $>2.5$ fold and RUNX3 by $>1.6$ fold, but no significant change in the expression of RASSF1 (Figure 6b). 5-Aza-CdR plus U-4149 produced an activation of CDKN1A by $>3.2$ fold and RASSF1 by $>2.5$ fold, but not RUNX3 as compared to the control cells (Figure $6 \mathrm{c}$ ). The activation of CDKN1A and RASSF1 by 5-Aza-CdR plus U-4149 was greater than the other combinations.

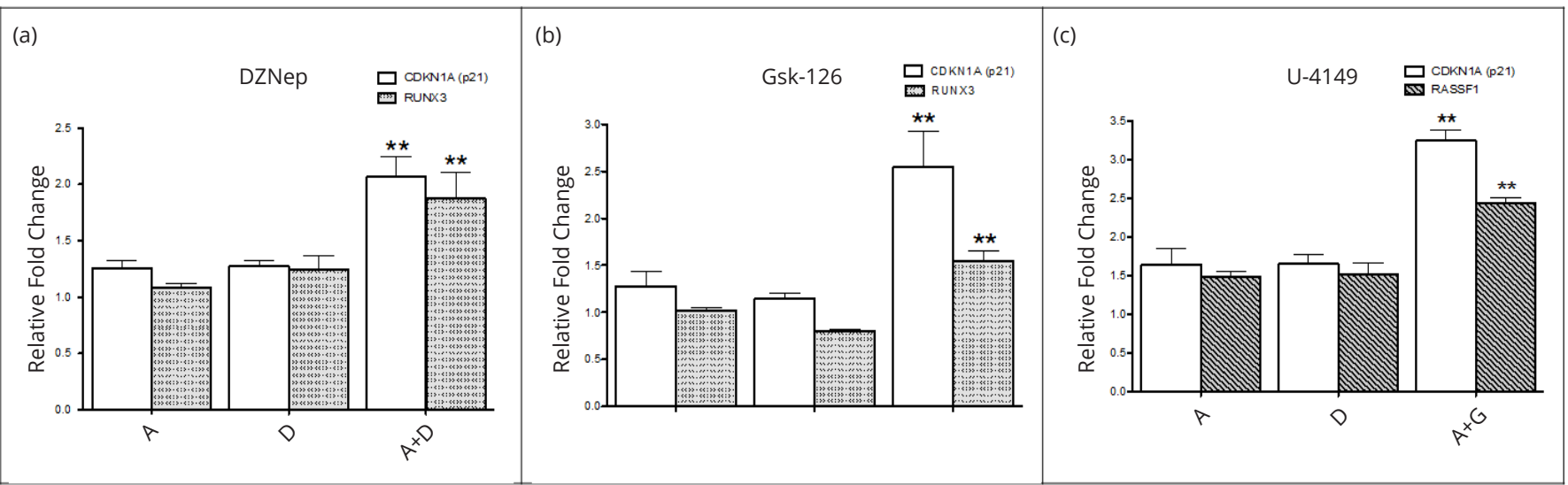

Figure $\mathbf{6 a}, \mathbf{b}, \mathbf{c}$ Real-time PCR analysis of gene expression in A549 lung cancer cells. Treatment is described in Table 1 . At $48 \mathrm{~h}$ the drugs were removed and at $72 \mathrm{~h}$ RNA was isolated for RT-PCR analysis as described in Methods. The relative gene expression is shown as mean \pm SEM, $\mathrm{n}=3$. Statistical analysis was made by one way ANOVA; ** $(P<0.05)$

Note: Wording within each graph very difficult to read. If possible should be enlargened.

\section{Discussion}

Aberrant DNA methylation was shown to be a key epigenetic modification of TSGs in NSCLC [30] making it an attractive target in cancer therapeutics. Inhibition of DNMT1 is currently being investigated for the therapy of NSCLC (ClinicalTrials.gov Identifier: NCT01935947). A phase I-II clinical trial on 5-Aza-CdR was performed at our University medical center in patients with stage IV metastatic NSCLC [14]. This pilot study showed some promising activity, including one patient that survived 7 years. The therapeutic effectiveness of 5-Aza-CdR against cancer may be limited by the fact that it only reactivates $30-40 \%$ of the genes silenced by DNA methylation [31]. In addition, 5-Aza-CdR has difficulty to activate DNA methylated genes if they contain the histone gene-silencing marker, H3K27me3 [18, 19].

In the present work, we demonstrate that it is possible to increase the effectiveness of 5-Aza-CdR against lung cancer by using it in combination with other epigenetic agents. We selected drugs that target directly or indirectly EZH2, a subunit of PRC2 that is responsible for the methylation of H3K27 to silence gene expression. The agents we selected were global histone methylation inhibitors, DZNep and its related analogue, U-4149, and the specific EZH2 inhibitor Gsk-126. All these histone methylation inhibitors when used in combination with 5-Aza-CdR showed a synergistic effect against A549 lung carcinoma cells. U-4149 was showed to be more potent than DZNep or Gsk-126 as a single agent and in combination with 5-Aza-CdR.

DZNep is a derivative of the antibiotic neplanocin-A. DZNep is not a direct EZH2 inhibitor, but rather a SAH-hydrolase inhibitor. Inhibition of this enzyme leads to an increase of the intracellular SAH concentration and reduction in the level of S-adenosylmethionine, which results in the inhibition of EZH2 [19]. DZNep was able to reactivate EZH2 target genes and induced apoptosis in different types of cancer cells $[25,26,32]$. DZNep, as a single agent, also showed a significant antineoplastic activity against NSCLC cells [27]. We reported previously that 5-Aza-CdR plus DZNep exhibited a synergistic interaction against leukemic cells [28]. In the present work, our data show that this combination is also synergistic against A549 NSCLC cells. Our results also indicate that epigenetic therapy that specifically targets both DNMT and EZH2 is very effective. The combination of U-4149 plus 5-Aza-CdR was more potent for treatment of NSCLC than the combinations containing either DZNep or Gsk-126. U-4149 is the fluoroadenosine analogue of DZNep.

Previous studies have indicated that methylation of specific genes resulting in epigenetic silencing occurs in lung cancer [9]. Inactivation of these genes favors tumorigenesis by suppressing differentiation, apoptosis and senescence. Cellular senescence is a tumor-suppressive mechanism that permanently prevents cells from malignant transformation [33]. We investigated the induction of senescence by these epigenetic agents by evaluation of inhibition of cell growth and induction of $\beta$-galactosidase expression. All the double combinations showed induction of senescence in A549 tumor cells. The combination 5-AzaCdR plus U-4149 exhibited the highest level of senescence as compared with the other combinations.

The induction of apoptosis is an important action of many anticancer agents. Caspases play an important role in the regulation and execution of apoptotic cell death [34]. We reported previously that 5 -Aza-CdR in combination with DZNep induces a synergistic induction of apoptosis in leukemia cells [35]. We observed that the combination of these epigenetic agents also induce apoptosis in lung carcinoma cells. 
The silencing of the expression TSGs by DNA and histone methylation can play a crucial role in malignant transformation to NSCLC [9]. In order to determine if this epigenetic gene-silencing mechanism is reversible, we treated A549 tumor cells with 5-Aza-CdR, DZNep, Gsk126 and U-4149 and analyzed the expression of three TSGs: CDKN1A (p21), RUNX3 and RASSF1. These genes can play an important role in the regulation of cell growth of NSCLC.

CDKN1A (p21) is a cyclin-dependent kinase inhibitor that is correlated with cell-cycle arrest that precedes terminal differentiation in a variety of tissues. In response to DNA damage, p53 induces CDKN1A expression, which induces cell cycle arrest at the G1 checkpoint [36]. Aberrant DNA methylation of CDKN1A has been observed in various malignancies, including lung cancer [37]. Zheng et al demonstrated that treatment with DNMT1 inhibitors enhanced CDKN1A levels [38]. In our study, all the three combinations of inhibitors of DNA and histone methylation exhibited a synergistic activation of CDKN1A.

RUNX3 is a downstream effector of the TGF- $\beta$ signaling pathway, and has critical roles in the regulation of cell death by apoptosis [39]. RUNX3 is frequently silenced by DNA hyper-methylation in many cancers, including lung cancer [39-41]. RUNX3 methylation correlates with clinical stage and degree of differentiation in NSCLC [42]. In our study the combinations of 5-Aza-CdR plus DZNep and 5-Aza-CdR plus Gsk-126 exhibited a synergistic activation of RUNX3. These results showing an increase of the expression of RUNX3 by these epigenetic agents are in accord with their increase induction of apoptosis.

RASSF1A shows a high frequency of decreased expression and increased promoter methylation in NSCLC [43]. RASSF1A methylation was associated with poor prognosis in NSCLC patients $[44,45]$. In our study only the combination of 5-Aza-CdR plus U-4149 showed a synergistic activation of RASSF1.

\section{Conclusion}

Our results suggest that a combination of inhibitors of DNA and histone methylation have interesting potential for the therapy of lung cancer. Additional studies on the in vivo activity of these combinations in animal tumor models of lung cancer will be helpful in the selection of the combination that may have the greatest potential for effective clinical treatment of lung cancer.

\section{Acknowledgments}

The research was supported by the Conselho Nacional de Desenvolvimento Científico e Tecnológico(CNPq/Programa Ciências sem Fronteiras/Brazil) and grant \#700795 from the Canadian Cancer Society Research Institute.

\section{Conflict of interest}

The authors declare no conflicts of interest.

\section{References}

[1] Siegel R, Ward E, Brawley O, Jemal A. Cancer statistics, 2011: The impact of eliminating socioeconomic and racial disparities on premature cancer deaths. CA Cancer J Clin. 2011; 61(4):212-236.
[2] Ferlay J, Shin HR, Bray F, Forman D, Mathers C, et al. Estimates of worldwide burden of cancer in 2008: GLOBOCAN 2008. Int J Cancer. 2010; 127(12):2893-2917.

[3] Field JK, Oudkerk M, Pedersen JH, Duffy SW. Prospects for population screening and diagnosis of lung cancer. Lancet. 2013; 382(9893):732741.

[4] Yoo CB, Jones PA. Epigenetic therapy of cancer: past, present and future. Nat Rev Drug Discov. 2006; 5(1):37-50.

[5] Jones PA, Baylin SB. The fundamental role of epigenetic events in cancer. Nat Rev Genet. 2002; 3(6):415-428.

[6] Sharma S, Kelly TK, Jones PA. Epigenetics in cancer. Carcinogenesis. 2010; 31(1):27-36.

[7] Teitz T, Wei T, Valentine MB, Vanin EF, Grenet J, et al. Caspase 8 is deleted or silenced preferentially in childhood neuroblastomas with amplification of MYCN. Nat Med. 2000; 6(5):529-535.

[8] Conway KE, McConnell BB, Bowring CE, Donald CD, Warren ST, et al. TMS1, a novel proapoptotic caspase recruitment domain protein, is a target of methylation-induced gene silencing in human breast cancers. Cancer Res. 2000; 60(22):6236-6242.

[9] Richards KL, Zhang B, Sun M, Dong W, Churchill J, et al. Methylation of the candidate biomarker TCF21 is very frequent across a spectrum of early-stage nonsmall cell lung cancers. Cancer. 2011; 117(3):606-17.

[10] Schrump DS. Targeting epigenetic mediators of gene expression in thoracic malignancies. Biochim Biophys Acta. 2012; 1819(7):836-845.

[11] Fang JY, Yang L, Zhu HY, Chen YX, Lu J, et al. 5-Aza-2'-deoxycitydine induces demethylation and up-regulates transcription of p16INK4A gene in human gastric cancer cell lines. Chin Med J (Engl). 2004; 117(1):99-103.

[12] Chu LC, Eberhart CG, Grossman SA, Herman JG. Epigenetic silencing of multiple genes in primary CNS lymphoma. Int J Cancer. 2006; 119(10):2487-2491.

[13] Juttermann R, Li E, Jaenisch R. Toxicity of 5-aza-2'-deoxycytidine to mammalian cells is mediated primarily by covalent trapping of DNA methyltransferase rather than DNA demethylation. Proc Natl Acad Sci U S A. 1994; 91(25):11797-11801.

[14] Momparler RL, Bouffard DY, Momparler LF, Dionne J, Belanger K, et al. Pilot phase I-II study on 5-aza-2'-deoxycytidine (Decitabine) in patients with metastatic lung cancer. Anticancer Drugs. 1997; 8(4):358-368.

[15] Juergens RA, Wrangle J, Vendetti FP, Murphy SC, Zhao M, et al. Combination epigenetic therapy has efficacy in patients with refractory advanced non-small cell lung cancer. Cancer discovery. 2011; 1(7):598607.

[16] Liu K, Huang W, Gao W, He W. Effect of combined 5-aza-2'deoxycytidine and cisplatin treatment on the P15 lung adenocarcinoma cell line. Oncol Lett. 2015; 9(5):2007-2012.

[17] Shang D, Liu Y, Matsui Y, Ito N, Nishiyama H, et al. Demethylating agent 5-aza-2'-deoxycytidine enhances susceptibility of bladder transitional cell carcinoma to Cisplatin. Urology. 2008; 71(6):1220-1225.

[18] Kondo Y, Shen L, Cheng AS, Ahmed S, Boumber Y, et al. Gene silencing in cancer by histone H3 lysine 27 trimethylation independent of promoter DNA methylation. Nat Genet. 2008; 40(6):741-750.

[19] Miranda TB, Cortez CC, Yoo CB, Liang G, Abe M, et al. DZNep is a global histone methylation inhibitor that reactivates developmental genes not silenced by DNA methylation. Mol Cancer Ther. 2009; 8(6):15791588.

[20] Takawa M, Masuda K, Kunizaki M, Daigo Y, Takagi K, et al. Validation of the histone methyltransferase EZH2 as a therapeutic target for various types of human cancer and as a prognostic marker. Cancer Sci. 2011; 102(7):1298-1305.

[21] Wagener N, Macher-Goeppinger S, Pritsch M, Husing J, Hoppe-Seyler K, et al. Enhancer of zeste homolog 2 (EZH2) expression is an independent prognostic factor in renal cell carcinoma. BMC cancer. 2010; 10:524.

[22] Matsukawa Y, Semba S, Kato H, Ito A, Yanagihara K, et al. Expression of the enhancer of zeste homolog 2 is correlated with poor prognosis in human gastric cancer. Cancer Sci. 2006; 97(6):484-491.

[23] Kikuchi J, Kinoshita I, Shimizu Y, Kikuchi E, Konishi J, et al. Distinctive expression of the polycomb group proteins Bmi1 polycomb ring finger oncogene and enhancer of zeste homolog 2 in nonsmall cell lung cancers and their clinical and clinicopathologic significance. cancer. 2010; 116(12): 3015-3024.

[24] Kemp CD, Rao M, Xi S, Inchauste S, Mani H, et al. Polycomb repressor complex-2 is a novel target for mesothelioma therapy. Clin Cancer Res. 2012; 18(1):77-90.

[25] Crea F, Hurt EM, Mathews LA, Cabarcas SM, Sun L, et al. Pharmacologic disruption of Polycomb Repressive Complex 2 inhibits tumorigenicity and tumor progression in prostate cancer. Mol Cancer. 2011; 10:40. 
[26] Hayden A, Johnson PW, Packham G, Crabb SJ. S-adenosylhomocysteine hydrolase inhibition by 3-deazaneplanocin A analogues induces anticancer effects in breast cancer cell lines and synergy with both histone deacetylase and HER2 inhibition. Breast Cancer Res Treat. 2011; 127(1):109-119.

[27] Kikuchi J, Takashina T, Kinoshita I, Kikuchi E, Shimizu Y, et al. Epigenetic therapy with 3-deazaneplanocin A, an inhibitor of the histone methyltransferase EZH2, inhibits growth of non-small cell lung cancer cells. Lung Cancer. 2012; 78(2):138-143.

[28] Momparler RL, Idaghdour Y, Marquez VE, Momparler LF. Synergistic antileukemic action of a combination of inhibitors of DNA methylation and histone methylation. Leuk Res. 2012; 36(8):1049-1054.

[29] Choi WJ, Moon HR, Kim HO, Yoo BN, Lee JA, et al. Preparative and stereoselective synthesis of the versatile intermediate for carbocyclic nucleosides: effects of the bulky protecting groups to enforce facial selectivity. J Org Chem. 2004; 69(7):2634-2636.

[30] Cameron EE, Bachman KE, Myohanen S, Herman JG, Baylin SB. Synergy of demethylation and histone deacetylase inhibition in the re-expression of genes silenced in cancer. Nat Genet. 1999; 21(1):103107.

[31] Raynal NJ, Si J, Taby RF, Gharibyan V, Ahmed S, et al. DNA methylation does not stably lock gene expression but instead serves as a molecular mark for gene silencing memory. Cancer res. 2012; 72(5):1170-1181.

[32] Hibino $S$, Saito $Y$, Muramatsu T, Otani A, Kasai $Y$, et al. Inhibitors of enhancer of zeste homolog 2 (EZH2) activate tumor-suppressor microRNAs in human cancer cells. Oncogenesis. 2014; 3:e104.

[33] Kuilman T, Michaloglou C, Mooi WJ, Peeper DS. The essence of senescence. Genes Dev. 2010; 24(22):2463-2479.

[34] Pop C, Salvesen GS. Human caspases: Activation, specificity, and regulation. J Biol Chem. 2009; 284(33):21777-21781.

[35] Momparler RL, Cote S, Momparler LF, Idaghdour Y. Epigenetic therapy of acute myeloid leukemia using 5-aza-2'-deoxycytidine (decitabine) in combination with inhibitors of histone methylation and deacetylation. Clin Epigenetics. 2014; 6(1):19.

[36] Gartel AL, Radhakrishnan SK. Lost in transcription: p21 repression, mechanisms, and consequences. Cancer Res. 2005; 65(10):39803985.

[37] Teramen $H$, Tsukuda K, Tanaka N, Ueno $T$, Kubo $T$, et al. Aberrant methylation of p21 gene in lung cancer and malignant pleural mesothelioma. Acta medica Okayama. 2011; 65(3):179-184.

[38] Zheng QH, Ma LW, Zhu WG, Zhang ZY, Tong TJ. p21Waf1/Cip1 plays a critical role in modulating senescence through changes of DNA methylation. J Cell Biochem. 2006; 98(5):1230-1248.

[39] Subramaniam MM, Chan JY, Yeoh KG, Quek T, Ito K, et al. Molecular pathology of RUNX3 in human carcinogenesis. Biochim Biophys Acta. 2009; 1796(2):315-331.

[40] Chung JH, Lee HJ, Kim BH, Cho NY, Kang GH. DNA methylation profile during multistage progression of pulmonary adenocarcinomas. Virchows Arch. 2011; 459(2):201-211.

[41] Lee YS, Lee JW, Jang JW, Chi XZ, Kim JH, et al. Runx3 inactivation is a crucial early event in the development of lung adenocarcinoma. Cancer Cell. 2013; 24(5):603-616.

[42] Yu GP, Ji Y, Chen GQ, Huang B, Shen K, et al. Application of RUNX3 gene promoter methylation in the diagnosis of non-small cell lung cancer. Oncol let. 2012; 3(1):159-162.

[43] Pastuszak-Lewandoska D, Kordiak J, Migdalska-Sek M, Czarnecka KH, Antczak A, et al. Quantitative analysis of mRNA expression levels and DNA methylation profiles of three neighboring genes: FUS1, NPRL2/ G21 and RASSF1A in non-small cell lung cancer patients. Respir Res. 2015; $16: 76$.

[44] Kim DH, Kim JS, Ji YI, Shim YM, Kim H, et al. Hypermethylation of RASSF1A promoter is associated with the age at starting smoking and a poor prognosis in primary non-small cell lung cancer. Cancer Res. 2003; 63(13):3743-3746.

[45] Yanagawa N, Tamura G, Oizumi H, Kanauchi N, Endoh M, et al. Promoter hypermethylation of RASSF1A and RUNX3 genes as an independent prognostic prediction marker in surgically resected non-small cell lung cancers. Lung Cancer. 2007; 58(1):131-138. 